\title{
On limit behavior of a solution to boundary value problem in a plane sector
}

\author{
Shaban H. Kutaiba | Vladimir B. Vasilyevil
}

Chair of Applied Mathematics and Computer Modeling, Belgorod State National Research University, Pobedy street 85, Belgorod, 308015, Russia

\section{Correspondence}

Vladimir B. Vasilyev, Chair of Applied Mathematics and Computer Modeling, Belgorod State National Research University, Pobedy street 85, Belgorod 308015, Russia.

Email: vladimir.b.vasilyev@gmail.com
We study a certain boundary value problem in Sobolev-Slobodetskii spaces with integral condition in a plane excluding a ray from origin. Using auxiliary problem in outside of a convex cone and the wave factorization concept, we construct a general solution and consider transfer to limit boundary value problem. It was shown that limit boundary value problem can be solvable only if the boundary function satisfies to a certain singular functional equation.

\section{KEYWORDS}

elliptic pseudo-differential equation, limit boundary value problem, wave factorization, general solution

MSC CLASSIFICATION

35S15; 47G30

\section{1 | INTRODUCTION}

Theory of pseudo-differential operators and equations on manifolds with a smooth boundary was very intensive which was developed in the second half quarter of the last century, ${ }^{1-3}$ and now, there are a lot of applications of the theory. ${ }^{4-6}$ Many papers and books are related to a theory of elliptic pseudo-differential operators and equations on nonsmooth manifolds or on manifolds with nonsmooth boundaries. ${ }^{7-14}$ According to the local principle to obtain Fredholm property for a general pseudo-differential operator, we need to study invertibility properties for model operators in so called canonical domains. One of such domains is a cone.

The authors develop a special approach to studying elliptic pseudo-differential equations on manifolds with a non-smooth boundary. Key point of the approach is studying a unique solvability for a model equation in a canonical domains. Such canonical domains can be a whole space $\mathbb{R}^{m}$, a half-space $\mathbb{R}_{+}^{m}=\left\{x \in \mathbb{R}^{m}: x=\left(x^{\prime}, x_{m}\right), x_{m}>0\right\}$ or a certain cone in $\mathbb{R}^{m}$. Some results in this direction are included in the books ${ }^{15,16}$ and papers, ${ }^{17-19}$ but now, some new results were obtained, ${ }^{15,20-24}$ and it permits developing the approach more explicitly. Moreover, some results ${ }^{25,26}$ can help to describe more complicated situations than ordinary $m$-dimensional cone in $\mathbb{R}^{m}$, namely, we would like to consider here the situation when starting cone degenerates into a cone of a lower dimension. We will start from two-dimensional case.

Outline of the paper is the following. We introduce boundary value problem with additional integral condition for a model elliptic pseudo-differential operator in a plane sector. For solving this problem, we describe functional spaces, operators, and a special factorization for an elliptic symbol. Further, we find a solution for the boundary value problem, and study conditions under which the solution exists for limit value of a cone.

\section{2 | STATEMENT OF THE PROBLEM AND AUXILIARIES}

Let $D$ be a plane domain of the following type $D=\mathbb{R}^{2} \backslash\left\{x \in \mathbb{R}^{2}: x_{1}=0, x_{2}>0\right\}$, and $A$ be an elliptic pseudo-differential operator with the symbol $A(\xi)^{3}$ satisfying the condition: 


$$
a_{1}(1+|\xi|)^{\alpha} \leq|A(\xi)| \leq a_{2}(1+|\xi|)^{\alpha},
$$

with positive constants $a_{1}, a_{2}$.

We will study the following boundary value problem

$$
\begin{aligned}
& (A u)(x)=v(x), \quad x \in D, \\
& \int_{-\infty}^{+\infty} u\left(x_{1}, x_{2}\right) d x_{2}=g\left(x_{1}\right) .
\end{aligned}
$$

Let us note that the condition (2) is the so-called nonlocal or integral condition. It appears in studies not often, but nevertheless, it is used in studying some problems. ${ }^{27-29}$

Our strategy is the following. Let $C_{+}^{a}=\left\{x \in \mathbb{R}^{2}: x=\left(x_{1}, x_{2}\right), x_{2}>a\left|x_{1}\right|, a>0\right\}$ be plane sector with the size $2 \alpha$ (so that $a=\cot \alpha$ ), and we will study Equation (1) with the condition (2) in the domain $\mathbb{R}^{2} \backslash C_{+}^{a}$. So our starting equation will be the following:

$$
(A u)(x)=v(x), \quad x \in \mathbb{R}^{2} \backslash C_{+}^{a} .
$$

Further, if we can find the solution of the problem (3), (2) then we will try to obtain limit expression for the solution under $a \rightarrow \infty(\alpha \rightarrow 0)$. We will see that under our assumptions the problem (1),(2) can be solvable only if the function $g$ satisfies a certain equation.

\section{1 | Spaces and operators}

We study Equation (3) in Sobolev-Slobodetskii space $H^{s}\left(\mathbb{R}^{2} \backslash C_{+}^{a}\right)$. By definition, this space consists of functions $u$ from $H^{s}\left(\mathbb{R}^{m}\right)$ which supports belong to $\overline{\mathbb{R}^{2} \backslash C_{+}^{a}}$. A norm in the space $H^{s}\left(\mathbb{R}^{2} \backslash C_{+}^{a}\right)$ is induced by the $H^{s}$-norm

$$
\|u\|_{s}=\left(\int_{\mathbb{R}^{3}} \tilde{u}(\xi)(1+|\xi|)^{2 s} d \xi\right)^{1 / 2}
$$

where the sign $\sim$ over $u$ denotes its Fourier transform

$$
\tilde{u}(\xi)=\int_{\mathbb{R}^{3}} u\left(x \left(e^{i x \cdot \xi} d x\right.\right.
$$

The right hand side $v$ in Equation (3) is taken from the space $H_{0}^{s-\alpha}\left(\mathbb{R}^{2} \backslash C_{+}^{a}\right)$ of functions defined in $\mathbb{R}^{2} \backslash C_{+}^{a}$ which admit a continuation $\ell v$ into whole $H^{s-\alpha}\left(\mathbb{R}^{m}\right)$. The norm in such a space is defined as

$$
\|v\|_{s}^{+}=\inf \|\ell v\|_{s}
$$

where inf is taken over all continuations $\ell v$.

Let us remind ${ }^{3}$ that a pseudo-differential operator $A$ is defined by its symbol $A(\xi)$ in the following way:

$$
(A u)(x)=\frac{1}{(2 \pi)^{m}} \int_{\mathbb{R}^{2}} e^{-i x \cdot \xi} \tilde{u}(\xi) d \xi .
$$

Generally speaking usually, they consider more general symbols $A(x, \xi)$ depending on a spatial variable $x$, but here, we will consider the simplest variant.

The operator $A$ with the symbol $A(\xi)$ satisfying the condition $(*)$ is a linear bounded operator $H^{s}\left(\mathbb{R}^{2} \backslash C_{+}^{a}\right) \rightarrow$ $H^{s-\alpha}\left(\mathbb{R}^{2} \backslash C_{+}^{a}\right) .^{3}$ 


\section{2 | Wave factorization}

Our study is based on a concept of the wave factorization. ${ }^{15,16}$ Before introducing the concept, we will remind some definitions from multidimensional analysis. ${ }^{30}$

If $C$ is a convex cone in $\mathbb{R}^{2}$, then the conjugate cone ${ }^{*}$ is defined as follows:

$$
\stackrel{*}{C}=\left\{x \in \mathbb{R}^{2}: x \cdot y=x_{1} y_{1}+x_{2} y_{2}>0, \quad \forall y \in C\right\} .
$$

Obviously, $C_{+}^{*}=\left\{x \in \mathbb{R}^{2}: a x_{2}>\left|x_{1}\right|\right\}$. Let us denote $C_{-}^{*}=-C_{+}^{*}$.

A radial tube domain over the cone $C$ is called a subset of two-dimensional complex space $\mathbb{C}^{2}$ of the following type:

$$
T(C)=\left\{z \in \mathbb{C}^{2}: z=x+i y, x \in \mathbb{R}^{2}, y \in C\right\} .
$$

Definition 1. The wave factorization of an elliptic symbol $A(\xi)$ with respect to the cone $C_{+}^{a}$ is called its representation in the form

$$
A(\xi)=A_{\neq}(\xi) A_{=}(\xi),
$$

where factors $A_{\neq}(\xi), A_{=}(\xi)$ must satisfy the following conditions:

1) $A_{\neq}(\xi), A_{=}(\xi)$ are defined for all $\xi \in \mathbb{R}^{2}$ may be except $\left\{\xi \in \mathbb{R}^{2}:\left|\xi_{1}\right|^{2}=a^{2} \xi_{2}^{2}\right\}$;

2) $A_{\neq}(\xi), A_{=}(\xi)$ admit an analytic continuation into radial tube domains $T\left(C_{-}^{*}\right), T\left(C_{+}^{a}\right)$ respectively with estimates

$$
\begin{gathered}
\left|A_{\neq}^{ \pm 1}(\xi+i \tau)\right| \leq c_{1}(1+|\xi|+|\tau|)^{ \pm æ}, \\
\left|A_{\equiv}^{ \pm 1}(\xi-i \tau)\right| \leq c_{2}(1+|\xi|+|\tau|)^{ \pm(\alpha-\mathfrak{x})}, \quad \forall \tau \in C_{-}^{*} .
\end{gathered}
$$

The number $æ \in \mathbb{R}$ is called an index of the wave factorization.

Remark 1. Let us note that we replace in the definition $C_{+}^{*}, C_{-}^{*}$ in a comparison with standard definition of the wave factorization. ${ }^{16}$

\subsection{A special integral operator $G_{2}$}

Let us define this operator in the following way ${ }^{16}$ first for functions $u$ from Schwartz space $S\left(\mathbb{R}^{2}\right)$

$$
\left(G_{2} \tilde{u}\right)\left(\xi_{1}, \xi_{2}\right)=\lim _{\tau \rightarrow 0+} \int_{\mathbb{R}^{2}} \frac{2 a \tilde{u}\left(\eta_{1}, \eta_{2}\right) d \eta}{\left(\xi_{1}-\eta_{1}\right)^{2}-a^{2}\left(\xi_{2}-\eta_{2}+i \tau\right)^{2}} .
$$

This operator plays an important role for constructing a solution of Equation (3). It is linear bounded operator $H^{s}\left(\mathbb{R}^{2} \rightarrow\right.$ $H^{s}\left(\mathbb{R}^{2}\right)$ for $|s|<1 / 2 .{ }^{16}$

If we denote by $\widetilde{H}^{s}\left(C_{+}^{a}\right), \widetilde{H}^{s}\left(\mathbb{R}^{2} \backslash C_{+}^{a}\right)$ the Fourier images of spaces $H^{s}\left(C_{+}^{a}\right), H^{s}\left(\mathbb{R}^{2} \backslash C_{+}^{a}\right)$, respectively, then an arbitrary function $\tilde{f} \in \widetilde{H}^{s}\left(\mathbb{R}^{2}\right.$ can be uniquely represented in the form

$$
\tilde{f}=\tilde{f}_{+}+\tilde{f}_{-}
$$

where $\tilde{f}_{+} \in \widetilde{H}^{s}\left(C_{+}^{a}\right), \tilde{f}_{-} \in \widetilde{H}^{s}\left(\mathbb{R}^{2} \backslash C_{+}^{a}\right)$ and

$$
\tilde{f}=G_{1} \tilde{f}+\left(I-G_{2}\right) \tilde{f}
$$

for $|s|<1 / 2$.

\section{4 | Transmutation operator}

Our further considerations are based on a special transmutation operator which is related to the Fourier transform. 
We introduce $T_{a}: \mathbb{R}^{2} \rightarrow \mathbb{R}^{2}$ of the following type:

$$
\left\{\begin{array}{r}
t_{1}=x_{1} \\
t_{2}=x_{2}-a\left|x_{1}\right|
\end{array}\right.
$$

This operator transforms $\partial C_{+}^{a}$ into hyperplane $x_{2}=0$.

We are interested in the operator $F T_{a} F^{-1}$; therefore, first, we need to study $F T_{1}$ and to find its explicit form. We have

$$
\begin{array}{r}
\left(F T_{a} u\right)(\xi)=\int_{-\infty}^{+\infty} e^{i a\left|y_{1}\right| \xi_{2}} e^{i y_{1} \xi_{1}} \hat{u}\left(y_{1}, \xi_{2}\right) d y_{1} \\
=\int_{-\infty}^{+\infty} \chi_{+}\left(y_{1}\right) e^{i a y_{1} \xi_{2}} e^{i y_{1} \xi_{1}} \hat{u}\left(y_{1}, \xi_{2}\right) d y_{1}+\int_{-\infty}^{+\infty} \chi_{-}\left(y_{1}\right) e^{-i a y_{1} \xi_{2}} e^{i y_{1} \xi_{1}} \hat{u}\left(y_{1}, \xi_{2}\right) d y_{1} \\
=\int_{-\infty}^{+\infty} \chi_{+}\left(y_{1}\right) e^{i y_{1}\left(a \xi_{2}+\xi_{1}\right)} \hat{u}\left(y_{1}, \xi_{2}\right) d y_{1}+\int_{-\infty}^{+\infty} \chi_{-}\left(y_{1}\right) e^{i y_{1}\left(-a \xi_{2}+\xi_{1}\right)} \hat{u}\left(y_{1}, \xi_{2}\right) d y_{1},
\end{array}
$$

where $\chi_{ \pm}$is an indicator of the half-axis $\mathbb{R}_{ \pm}$.

The last two summands are the Fourier transforms of functions

$$
\chi_{+}\left(y_{1}\right) e^{i y_{1}\left(a \xi_{2}+\xi_{1}\right)} \hat{u}\left(y_{1}, \xi_{2}\right), \quad \chi_{-}\left(y_{1}\right) e^{i y_{1}\left(-a \xi_{2}+\xi_{1}\right)} \hat{u}\left(y_{1}, \xi_{2}\right),
$$

on the first variable $y_{1}$, respectively, so we can use Plemelj-Sokhotskii formulas ${ }^{31-33}$ (see also Eskin ${ }^{3}$ ), and we write them as follows:

$$
\begin{aligned}
& \int_{-\infty}^{+\infty} \chi_{+}(x) e^{i x \xi} u(x) d x=\frac{1}{2} \tilde{u}(\xi)+v \cdot p \cdot \frac{i}{2 \pi} \int_{-\infty}^{+\infty} \frac{\tilde{u}(\eta) d \eta}{\xi-\eta}, \\
& \int_{-\infty}^{+\infty} \chi_{-}(x) e^{i x \xi} u(x) d x=\frac{1}{2} \tilde{u}(\xi)-v \cdot p \cdot \frac{i}{2 \pi} \int_{-\infty}^{+\infty} \frac{\tilde{u}(\eta) d \eta}{\xi-\eta},
\end{aligned}
$$

where v.p. denotes principal value of the integral in Cauchy sense. ${ }^{31}$

Thus, we obtain

Let us denote

$$
\begin{gathered}
\left(F T_{a} u\right)(\xi)=\frac{\tilde{u}\left(\xi_{1}+a \xi_{2}, \xi_{2}\right)+\tilde{u}\left(\xi_{1}-a \xi_{2}, \xi_{2}\right)}{2} \\
+v \cdot p \cdot \frac{i}{2 \pi} \int_{-\infty}^{+\infty} \frac{\tilde{u}\left(\eta, \xi_{2}\right) d \eta}{\xi_{1}+a \xi_{2}-\eta}-v \cdot p \cdot \frac{i}{2 \pi} \int_{-\infty}^{+\infty} \frac{\tilde{u}\left(\eta, \xi_{2}\right) d \eta}{\xi_{1}-a \xi_{2}-\eta} .
\end{gathered}
$$

where

$$
P_{1}=\frac{1}{2}\left(I+S_{1}\right), \quad Q_{1}=\frac{1}{2}\left(I-S_{1}\right),
$$

then we can write

$$
\left(S_{1} \tilde{u}\right)\left(\xi_{1}, \xi_{2}\right)=\frac{i}{\pi} v \cdot p \cdot \int_{-\infty}^{+\infty} \frac{\tilde{u}\left(\eta, \xi_{2}\right) d \eta}{\xi_{1}-\eta}
$$

$$
\left(F T_{a} u\right)\left(\xi_{1}, \xi_{2}\right)=\left(P_{1} \tilde{u}\right)\left(\xi_{1}+a \xi_{2}, \xi_{2}\right)+\left(Q_{1} \tilde{u}\right)\left(\xi_{1}-a \xi_{2}, \xi_{2}\right)
$$

Corollary 1. If

then

$$
u\left(x_{1}, x_{2}\right)=\sum_{k=0}^{n-1} c_{k}\left(x_{1}\right) \delta^{(k)}\left(x_{2}\right)
$$

$$
\left(F T_{a} u\right)\left(\xi_{1}, \xi_{2}\right)=\sum_{k=0}^{n-1} \xi_{2}^{k}\left(\left(P_{1} \tilde{c}_{k}\right)\left(\xi_{1}+a \xi_{2}\right)+\left(Q_{1} \tilde{c}_{k}\right)\left(\xi_{1}-a \xi_{2}\right)\right)
$$




\section{3 | A GENERAL SOLUTION}

If the symbol $A(\xi)$ admits the wave factorization ${ }^{16}$ under the condition $1 / 2<\alpha-s<3 / 2$, where $\alpha$ is the index of wave factorization, then one can show ${ }^{34}$ that a general solution of the homogeneous Equation (3) in Sobolev-Slobodetskii space $H^{s}\left(C_{+}^{a}\right)$ in Fourier image has the following form:

$$
\begin{array}{r}
\tilde{u}(\xi)=\frac{\tilde{c}_{0}\left(\xi_{1}+a \xi_{2}\right)+\tilde{c}_{0}\left(\xi_{1}-a \xi_{2}\right)}{2 A_{\neq}\left(\xi_{1}, \xi_{2}\right)}+ \\
A_{\neq}^{-1}\left(\xi_{1}, \xi_{2}\right)\left(v \cdot p \cdot \frac{i}{2 \pi} \int_{-\infty}^{+\infty} \frac{\tilde{c}_{0}(\eta) d \eta}{\xi_{1}+a \xi_{2}-\eta}-v \cdot p \cdot \frac{i}{2 \pi} \int_{-\infty}^{+\infty} \frac{\tilde{c}_{0}(\eta) d \eta}{\xi_{1}-a \xi_{2}-\eta}\right),
\end{array}
$$

where $c_{0}$ is an arbitrary function from $H^{s-æ+1 / 2}(\mathbb{R})$.

Here, we will consider Equation (3) for the case $æ-s=n+\delta, n \in \mathbb{N},|\delta|<1 / 2$ for the cone $\mathbb{R}^{2} \backslash C_{+}^{a}$.

Theorem 1. Let the symbol $A(\xi)$ satisfies the condition (*) and admits the wave factorization with respect to the cone $C_{+}^{a}$ with the index $\propto, \propto-s=n+\delta, n \in \mathbb{N},|\delta|<1 / 2$. A general solution of Equation (3) in Fourier image is given by the formula

$$
\tilde{u}(\xi)=A_{\neq}^{-1}(\xi) Q_{n}(\xi)\left(I-G_{2}\right) Q_{n}^{-1}(\xi) A_{=}^{-1}(\xi) \tilde{\ell} v(\xi)+A_{\neq}^{-1}(\xi) F T_{-a} F^{-1}\left(\sum_{k=0}^{n-1} \tilde{c}_{k}\left(\xi_{1}\right) \xi_{2}^{k}\right),
$$

where $_{k}\left(x^{\prime}\right) \in H^{s_{k}}\left(\mathbb{R}^{m-1}\right)$ are arbitrary functions, $s_{k}=s-a+k+1 / 2, k=0,1,2, \ldots, n-1, \ell v$ is an arbitrary continuation off on $H^{s-\alpha}\left(\mathbb{R}^{m}\right), Q_{n}(\xi)$ is an arbitrary polynomial satisfying the condition (*) for $\alpha=n$.

Proof. After wave factorization for the symbol with preliminary Fourier transform, we write

$$
A_{\neq}(\xi) \tilde{u}(\xi)+A_{=}^{-1}(\xi) \tilde{u}_{-}(\xi)=A_{=}^{-1}(\xi) \tilde{\ell} v(\xi),
$$

where $u_{-}(x)=\ell v(x)-u(x), \ell v$ is an arbitrary continuation of $v$ on the whole $\mathbb{R}^{2}$.

One can see that $A_{=}^{-1}(\xi) \tilde{\ell} v(\xi)$ belongs to the space $\tilde{H}^{s-x}\left(\mathbb{R}^{2}\right)$, and if we choose the polynomial $Q_{n}(\xi)$, satisfying the condition

$$
\left|Q_{n}(\xi)\right| \sim(1+|\xi|)^{n},
$$

then $Q_{n}^{-1}(\xi) A_{=}^{-1}(\xi) \tilde{\ell} v(\xi)$ will belong to the space $\tilde{H}^{-\delta}\left(\mathbb{R}^{2}\right)$.

Further, according to the theory of multi-dimensional Riemann problem, ${ }^{16}$ we can decompose the last function on two summands (jump problem):

$$
Q_{n}^{-1} A_{=}^{-1} \tilde{\ell} v=f_{+}+f_{-},
$$

where $f_{+} \in \tilde{H}\left(C_{+}^{a}\right), f_{-} \in \tilde{H}\left(\mathbb{R}^{2} \backslash C_{+}^{a}\right)$, and

$$
f_{+}=\left(I-G_{2}\right)\left(Q_{n}^{-1} A_{=}^{-1} \tilde{\ell} v\right), \quad f_{-}=G_{2}\left(Q_{n}^{-1} A_{=}^{-1} \tilde{\ell} v\right) .
$$

Therefore, we obtain

$$
Q_{n}^{-1} A_{\neq} \tilde{u}+Q_{n}^{-1} A_{=}^{-1} \tilde{u}_{-}=f_{+}+f_{-},
$$

or

$$
Q_{n}^{-1} A_{\neq} \tilde{u}-f_{+}=f_{-}-Q_{n}^{-1} A_{=}^{-1} \tilde{u}_{-} .
$$

Rewriting we have

$$
A_{\neq} \tilde{u}-Q_{n} f_{+}=Q_{n} f_{-}-A_{=}^{-1} \tilde{u}_{-} .
$$

The left-hand side of the equality belongs to the space $\tilde{H}^{-n-\delta}\left(\mathbb{R}^{2} \backslash C_{+}^{a}\right)$, and the right-hand side belongs to $\tilde{H}^{-n-\delta}\left(C_{+}^{a}\right)$. Hence,

$$
F^{-1}\left(A_{\neq} \tilde{u}-Q f_{+}\right)=F^{-1}\left(Q f_{-}-A_{=}^{-1} \tilde{u}_{-}\right),
$$

where the left-hand side belongs to $H^{-n-\delta}\left(\mathbb{R}^{2} \backslash C_{+}^{a}\right)$, and right-hand side belongs to $H^{-1-\delta}\left(C_{+}^{a}\right)$; therefore, we conclude immediately that this is a distribution supported on $\partial C_{+}^{a}$. 
Taking into account a general form for a distribution from $S^{\prime}\left(\mathbb{R}^{2}\right)$ supported on the straight line $x_{2}=0^{3,30}$

$$
c\left(x_{1}, x_{2}\right)=\sum_{k=0}^{m} c_{k}\left(x_{1}\right) \delta^{(k)}\left(x_{2}\right)
$$

we need to apply the transform $T_{-a}$ to the formula (6)to obtain the distribution supported on $\partial C_{+}^{a}$.

The formula (6) in the fourier image looks as follows:

$$
\tilde{c}\left(\xi_{1}, \xi_{2}\right)=\sum_{k=0}^{m} \tilde{c}_{k}(\xi 1) \xi_{2}^{k}
$$

Because such distribution should be belonging to $\tilde{H}^{-n-\delta}\left(\mathbb{R}^{2}\right)$, we need to estimate the integrals

$$
\begin{aligned}
& \int_{\mathbb{R}^{2}}\left|\tilde{c}_{k}\left(\xi_{1}\right)\right|^{2}\left|\xi_{2}\right|^{2 k}(1+|\xi|)^{2(-n-\delta)} d \xi=\int_{\mathbb{R}^{2}}\left|\tilde{c}_{k}\left(\xi_{1}\right)\right|^{2}\left|\xi_{2}\right|^{2 k}(1+|\xi|)^{2(s-\mathfrak{x})} d \xi \leq \\
& \text { const } \int_{\mathbb{R}^{2}}\left|\tilde{c}_{k}\left(\xi_{1}\right)\right|^{2}(1+|\xi|)^{2(k+s-æ)} d \xi=\text { const } \int_{-\infty}^{+\infty}\left|\tilde{c}_{k}\left(\xi_{1}\right)\right|^{2}\left(\int_{-\infty}^{+\infty}\left(1+\left|\xi_{1}\right|+\left|\xi_{2}\right|\right)^{2(k+s-æ)} d \xi_{2}\right) d \xi_{1} .
\end{aligned}
$$

The latter inner integral converges only if

$$
2(k+s-æ)<-1 .
$$

If the condition (7) is valid, then by integrating on $\xi_{2}$, we obtain

$$
\int_{\mathbb{R}^{2}}\left|\tilde{c}_{k}\left(\xi_{1}\right)\right|^{2}\left|\xi_{2}\right|^{2 k}(1+|\xi|)^{2(-n-\delta)} d \xi \leq \mathrm{const} \int_{-\infty}^{+\infty}\left|\tilde{c}_{k}\left(\xi_{1}\right)\right|^{2}\left(1+\left|\xi_{1}\right|\right)^{2(k+s-æ+1 / 2)} d \xi_{1}
$$

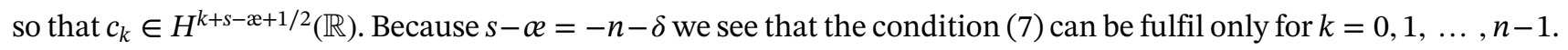

Thus, we have exactly $n$ summands in the formula (6), that is, $m=n-1$.

Now, in equality (5), we will write as follows:

$$
F^{-1}\left(A_{\neq} \tilde{u}-Q f_{+}\right)=T_{-a} c .
$$

Further, applying the Fourier transform $F$ to both left and hand side of the latter formula, we obtain the formula (4).

Remark 2. According to Corollary 1 , it is obvious that

$$
\left(F T_{-a} c\right)\left(\xi_{1}, \xi_{2}\right)=\sum_{k=0}^{n-1} \xi_{2}^{k}\left(\left(Q_{1} \tilde{c}_{k}\right)\left(\xi_{1}+a \xi_{2}\right)+\left(P_{1} \tilde{c}_{k}\right)\left(\xi_{1}-a \xi_{2}\right)\right)
$$

Corollary 2. If $a \rightarrow \infty$ then a general solution of the equation of Equation (1) depends on unique function $c_{0}\left(x_{1}\right)$.

Proof. According to Corollary 1, we have

$$
\left(F T_{a} u\right)\left(\xi_{1}, \xi_{2}\right)=\sum_{k=0}^{n-1} \xi_{2}^{k}\left(\left(P_{1} \tilde{c}_{k}\right)\left(\xi_{1}+a \xi_{2}\right)+\left(Q_{1} \tilde{c}_{k}\right)\left(\xi_{1}-a \xi_{2}\right)\right) .
$$

Let us make the change of variables 


$$
\left\{\begin{array}{l}
t_{1}=\xi_{1}+a \xi_{2} \\
t_{2}=\xi_{1}-a \xi_{2}
\end{array}\right.
$$

Then, we obtain

$$
\begin{aligned}
\left(F T_{a} u\right)\left(\frac{t_{1}+t_{2}}{2}, \frac{t_{1}-t_{2}}{2 a}\right)= & \left.P_{1} \tilde{c}_{0}\right)\left(t_{1}\right)+\left(Q_{1} \tilde{c}_{0}\right)\left(t_{2}\right)+ \\
& +\sum_{k=1}^{n-1}\left(\frac{t_{1}-t_{2}}{2 a}\right)^{k}\left(\left(P_{1} \tilde{c}_{k}\right)\left(t_{1}\right)+\left(Q_{1} \tilde{c}_{k}\right)\left(t_{2}\right)\right),
\end{aligned}
$$

so we see that under $a \rightarrow \infty$ the limit exists for arbitrary fixed collection $\left\{\tilde{c}_{k}\right\}_{k=1}^{n-1}$.

Therefore, we conclude that for studying the limit boundary value problem under $a \rightarrow \infty$ we need to determine only one arbitrary function $c_{0}$.

\section{4 | BOUNDARY VALUE PROBLEMS}

Let us denote $\tilde{u}(\xi)=A_{\neq}^{-1}(\xi) Q_{n}(\xi)\left(I-G_{2}\right) Q_{n}^{-1}(\xi) A_{=}^{-1}(\xi) \tilde{\ell} v(\xi) \equiv \tilde{f}$. Then according to Theorem 1 and Remark 2, we have the following formula for a general solution of Equation (3):

$$
\tilde{u}(\xi)=\tilde{f}(\xi)+A_{\neq}^{-1}(\xi) \sum_{k=0}^{n-1} \xi_{2}^{k}\left(\left(Q_{1} \tilde{c}_{k}\right)\left(\xi_{1}+a \xi_{2}\right)+\left(P_{1} \tilde{c}_{k}\right)\left(\xi_{1}-a \xi_{2}\right)\right) .
$$

Taking into account that the condition (2) in Fourier image takes the form,

$$
\tilde{u}\left(\xi_{2}, 0\right)=\tilde{g}\left(\xi_{1}\right)
$$

and substituting it into the formula (8), we obtain

$$
\tilde{u}\left(\xi_{1}, 0\right)=\tilde{g}\left(\xi_{1}\right)=\tilde{f}\left(\xi_{1}, 0\right)+A_{\neq}^{-1}\left(\xi_{1}, 0\right) \tilde{c}_{0}\left(\xi_{1}\right) .
$$

Therefore, we can find $\tilde{c}_{0}$

$$
\tilde{c}_{0}\left(\xi_{1}\right)=\left(\tilde{g}\left(\xi_{1}\right)-\tilde{f}\left(\xi_{1}, 0\right)\right) A_{\neq}\left(\xi_{1}, 0\right) .
$$

\section{1 | The case $\boldsymbol{v} \equiv 0$}

For this case, the formula (8) reduces to the following:

$$
\tilde{u}(\xi)=A_{\neq}^{-1}(\xi) \sum_{k=0}^{n-1} \xi_{2}^{k}\left(\left(Q_{1} \tilde{c}_{k}\right)\left(\xi_{1}+a \xi_{2}\right)+\left(P_{1} \tilde{c}_{k}\right)\left(\xi_{1}-a \xi_{2}\right)\right)
$$

and formula for $\tilde{c}_{0}$ looks as follows:

$$
\tilde{c}_{0}\left(\xi_{1}\right)=\tilde{g}\left(\xi_{1}\right) A_{\neq}\left(\xi_{1}, 0\right) .
$$

We make change of variables in the formula (9) like the proof of Corollary 2 and obtain

$$
\tilde{u}\left(\frac{t_{1}+t_{2}}{2}, \frac{t_{1}-t_{2}}{2 a}\right)=A_{\neq}^{-1}\left(\frac{t_{1}+t_{2}}{2}, \frac{t_{1}-t_{2}}{2 a}\right) \sum_{k=0}^{n-1}\left(\frac{t_{1}-t_{2}}{2 a}\right)^{k}\left(\left(Q_{1} \tilde{c}_{k}\right)\left(t_{1}\right)+\left(P_{1} \tilde{c}_{k}\right)\left(t_{2}\right)\right) .
$$

Then we see that under $a \rightarrow \infty$, the following equality,

$$
u\left(\frac{t_{1}+t_{2}}{2}, 0\right)=A_{\neq}^{-1}\left(\frac{t_{1}+t_{2}}{2}, 0\right)\left(\left(Q_{1} \tilde{c}_{0}\right)\left(t_{1}\right)+\left(P_{1} \tilde{c}_{0}\right)\left(t_{2}\right)\right),
$$

appears. 


\section{5 | SOLVABILITY CONDITION}

Now, we are able to make a certain conclusion on solvability of starting boundary value problem (1),(2).

Let us denote

$$
\tilde{b}(t)=A_{\neq}(t, 0) .
$$

Taking into account our additional condition (2), we can write

$$
(\tilde{b} \tilde{g})\left(\frac{t_{1}+t_{2}}{2}\right)=\left(Q_{1}(\tilde{b} \tilde{g})\right)\left(t_{1}\right)+\left(P_{1}(\tilde{b} \tilde{g})\right)\left(t_{2}\right) .
$$

Theorem 2. Let elliptic symbol $A(\xi)$ admits wave factorization with respect to $C_{+}^{a}$ with index $\propto$ such that $\propto-s=n+\delta, n \in$ $\mathbb{N},|\delta|<1 / 2$ for all enough large $a$, and $v \equiv 0, g \in H^{s+1 / 2}(\mathbb{R})$. Then the limit problem (1),(2) can be solvable in the space $H^{s}(D)$ if and only if the function $g$ satisfies Equation (11) for all $t_{1}, t_{2}$.

Proof. We act like previous steps. First, we find a general solution (8) using wave factorization method. Second, we verify that limit of a general solution under $a \rightarrow \infty$ includes only one arbitrary function, which can be found using the condition (2). Third, changing variables and passing to limit we obtain Equation (11).

\section{6 | CONCLUSION}

As it was shown for limit boundary value problem, the value of index of wave factorization does not play such important role in comparison with standard case of a cone. Although there are a lot of solutions to preliminary boundary value problem, we have only one limit solution, and the solvability condition for considered limit boundary value problem is the same like the case $n=1$. Maybe if we will consider other types of additional conditions to determine arbitrary functions in a general solution, we will not find such a phenomenon.

\section{CONFLICTS OF INTEREST}

This work does not have any conflicts of interest.

\section{ORCID}

Vladimir B. Vasilyev (D) https://orcid.org/0000-0001-9351-8084

\section{REFERENCES}

1. Hörmander L. Analysis of Partial Differential Operators, Vol. I-IV. Berlin: Springer-Verlag; 1983.

2. Rempel S, Schulze B-W. Index Theory of Elliptic Boundary Problems. Berlin: Akademie-Verlag; 1982.

3. Eskin GI. Boundary Value Problems for Elliptic Pseudodifferential Equations. Providence: AMS; 1981.

4. Operator Theory, Pseudo-Differential Equations, and Mathematical Physics. Eds. Karlovich Y.I., Rodino L.G., Silbermann B. Basel: Birkhäuser/Springer, 2013.

5. Pseudo-Differential Operators: Partial Differential Equations and Time-Frequency Analysis (Fields Institute Communications). Eds. Rodino L., Schulze B.-W., Wong M.W. Providence, AMS, 2007.

6. Pseudo-Differential Operators: Quantization and Signals. Eds. Rodino L., Wong M.W. Lect. Notes Math. 2008. V. 1949.

7. Nazarov SA, Plamenevsky BA. Elliptic Problems in Domains with Piecewise Smooth Boundaries. Berlin-New York: Walter de Gruyter; 1994.

8. Schulze B-W, Sternin B, Shatalov V. Differential Equations on Singular Manifolds: Semiclassical Theory and Operator Algebras. Berlin: Wiley-VCH; 1998.

9. Nazaikinskii VE, Savin AY, Schulze B-W, Sternin BY. Elliptic Theory on Singular Manifolds. Boca Raton: Chapman \& Hall/CRC; 2006.

10. Nazaikinskii V, Schulze B-W, Sternin B. The Localization Problem in Index Theory of Elliptic Operators. Pseudo-Differential Operators Theory and Applications, Vol. 10. Basel: Birkhäuser/Springer; 2014.

11. Kottke C, Melrose RB. Generalized blow-up of corners and fiber products. Trans Am Math Soc. 2015;367(1):651-705.

12. Egorov JV, Schulze B-W. Pseudo-Differential Operators, Singularities, Applications. Basel: Birkhäuser-Verlag; 1997.

13. Plamenevskii B. Algebras of Pseudodofferential Operators. Netherlands: Springer; 1989.

14. Schulze B-W. Boundary Value Problems and Singular Pseudo-Differential Operators. Chichester: J. Wiley; 1998. 
15. Vasilyev VB. Multipliers of Fourier Integrals, Pseudodifferential Equations, the Wave Factorization, Boundary Value Problems. 2nd ed. Moscow: Editorial URSS; 2010. (Russian).

16. Vasil'ev VB. Wave Factorization of Elliptic Symbols: Theory and Applications. Dordrecht-Boston-London: Kluwer Academic Publishers; 2000.

17. Vasilyev VB. Elliptic equations and boundary value problems in non-smooth domains. In: Rodino L. et al., eds. Pseudo differential operators: Analysis, applications and computations. Basel: Birkhäuser:105-121.

18. Vasilyev VB. General boundary value problems for pseudo-differential equations and related difference equations. Adv Difference Equ. 2013;289:1-7.

19. Vasilyev VB. Elliptic equations, manifolds with non-smooth boundaries, and boundary value problems. In: Dang P, ed. New trends in analysis and interdisciplinary applications. Birkhäuser: Cham; 2017:337-344.

20. Vasilyev VB. Pseudo differential equations on manifolds with non-smooth boundaries. Differential and difference equations and applications, Vol. 47: Springer Proc. Math. \& Stat.; 2013:625-637.

21. Vasilyev VB. Potentials for elliptic boundary value problems in cones. Siberian Electron Math Repts 2016;13:1129-1149. (Russian).

22. Vasilyev VB. On certain elliptic problems for pseudo differential equations in a polyhedral cone. Adv Dyn Syst Appl. 2014;9(2):227-237.

23. Vasilyev VB. New constructions in the theory of elliptic boundary value problems. Integral methods in science and engineering. Proc. IMSE conference, karlsruhe, germany, 2014. Basel: Birkhäuser; 2015:629-641.

24. Vasilyev VB. On the Dirichlet and Neumann problems in multi-dimensional cone. Math Bohem. 2014;139(2):333-340.

25. Vasilyev VB. Asymptotical analysis of singularities for pseudo differential equations in canonical non-smooth domains. Integral methods in science and engineering. Computational and analytic aspects. Boston: Birkhäuser; 2011:379-390.

26. Vasilyev VB. On the asymptotic expansion of certain plane singular integral operators. Bound Value Probl. 2017;116:1-13.

27. Avalishvili G, Avalishvili M, Gordeziani D. On a nonlocal problem with integral boundary conditions for a multidimensional elliptic equation. Appl Math Lett. 2011;24:566-571.

28. Egorov IE. Vragov boundary value problem with integral boundary condition for a mixed type equation. AIP Conf Proc. 2010;2172:030005.

29. Pulkina L. Nonlocal problems for hyperbolic equations from the viewpoint of strongly regular boundary conditions. Electron J Differ Equations. 2020;28:1-20.

30. Vladimirov VS. Generalized Functions in Mathematical Physics. Moscow: Mir; 1979.

31. Milkhin SG, Prößdorf S. Singular Integral Operators. Berlin: Akademie-Verlag; 1986.

32. Gakhov FD. Boundary Value Problems. NY: Dover Publications; 1981.

33. Muskhelishvili NI. Singular Integral Equations. North Holland Amsterdam; 1976.

34. Vasilyev VB. Pseudo-differential equations and conical potentials: 2-dimensional case. Opusc Math. 2019;39:109-124.

How to cite this article: Vasilyev VB, Kutaiba S. On limit behavior of a solution to boundary value problem in a plane sector. Math Meth Appl Sci. 2021;44:11904-11912. https://doi.org/10.1002/mma.6741 